\title{
Numerical Investigation of Unsteady Cavitation Flow around E779A Propeller in a Nonuniform Wake with an Insight on How Cavitation Influences Vortex
}

\author{
Chengzao Han, Yun Long, Xiaorui Bai, and Bin Ji \\ State Key Lab of Water Resources and Hydropower Engineering Science, Wuhan University, Wuhan 430072, China \\ Correspondence should be addressed to Bin Ji; jibin@whu.edu.cn
}

Received 13 January 2021; Revised 7 February 2021; Accepted 15 February 2021; Published 20 February 2021

Academic Editor: Ling Zhou

Copyright ( 2021 Chengzao Han et al. This is an open access article distributed under the Creative Commons Attribution License, which permits unrestricted use, distribution, and reproduction in any medium, provided the original work is properly cited.

\begin{abstract}
In the current study, the turbulent cavitation flow around a marine propeller in a nonuniform wake is simulated with the shear stress transport $(k-\omega S S T)$ turbulence model combining Zwart-Gerber-Belamri (ZGB) cavitation model. The predicted cavity evolution shows a fairly well agreement with the available experimental results. Important mechanisms of propeller cavitation flow, including side-entrant jet and cavitation-vortex interaction, are analyzed in this paper. Vorticity is found to be mainly located in cavitation regions and the propeller wake during propeller rotating. The unsteady behavior of cavitation and sideentrant jet can both promote local vorticity generation and flow unsteadiness. In addition, it is indicated with the relative vorticity transport equation that the stretching term plays a major role in vorticity transportation, while baroclinic torque and Coriolis force term mainly influence the vorticity distribution along the liquid-vapor interface.
\end{abstract}

\section{Introduction}

Cavitation is responsible for most considerable constraints in propeller and hydraulic machinery, related to noise, vibration, and pressure fluctuation. Moreover, it is also an unsteady phenomenon not yet neither effectively assessable nor fully understood. In order to reduce these undesirable effects, enormous effort has been put into investigations of the mechanisms behind cavitation turbulent flow experimentally and numerically.

The propeller motion through velocity discontinuous region causes a regular variation of the hydrostatic pressure on the blade surface; thus, the blade thrust and torque and cavitation performance are affected. This situation creates the necessary form of conditions favoring the cavitation generation and promotes the occurrence of unsteady cavitation on the blades, further enhanced by cavitation and vortex structure interaction. For marine propellers, turbulent cavitation evolution causes strong pressure pulsations radiating through the water in the near field or to the ship hull structure and then translating into hull vibrations and noise. The side effect generated by complex cavitation flow makes it the major source of propeller performance degradation, noise, and structural vibrations on a ship, as it contributes to compromise the stealth capabilities and stability of a surface or underwater warship.

Abundant literatures are available on the subject. Many researchers have carried out investigations to analyze the propeller performance with experiments so far. Alves Pereira et al. [1] made a quantitative analysis between the near pressure field variation and the cavitation evolution on the propeller blades. They used the harmonic analysis and image processing techniques to establish a correlation between the pressure fluctuation and cavitation pattern. Ito [2] investigated the cavitation flow around a propeller in nonuniform inflow based on the unsteady cavitation on an oscillating blade with different angles of attack. Bark and Van Berlekom [3] performed some experiments in a cavitation tunnel about the cavitation dynamics, radiated noise around an oscillating hydrofoil, and further discussed the relationship between them. Stella et al. [4] used two-phase sampling techniques to study cavitation flow around the propeller by means of LDV. 
The two implemented methods complement each other from the accuracy and efficiency aspects. Andrea et al. [5] compared and investigated the inflow characteristics and the separation mechanisms behind the ship hull with and without the propeller. They proposed that the separated flow region extension in the stern can be accurately identified by using the skewness coefficient. Lee et al. [6] used SPIV technique to measure all three velocity components of a propeller wake at different advance ratios. The results showed that the accurate analysis of the wake field is significant for the propeller design. Felli et al. [7] also measured the velocity and pressure fields behind a marine propeller based on the PIV technique to investigate the propeller wake flow. The analysis showed the correlation between the vorticity evolution and pressure fluctuation.

Experimental observations can obtain many of the intuitive phenomena but suffers from some limitations in the measurement techniques. A typical example is in measuring internal flow and vortical structures, which may hide themselves when using current optical measurement techniques due to cavitation, but are often important for finding the source of structural vibration. The access to the complete and refined flow field through a numerical simulation method would thus be an effective complement to experimental data. Lindau et al. [8] simulated the cavitation flow around a propeller in different advance ratios and compared with the experiments. Their simulations were performed validating against water tunnel measured thrust and torque breakdown for the propeller. Cai et al. [9] investigated and evaluated the capability of different turbulence models, including SRS, RANS, DLES, and DDES, in calculating the propeller hydrodynamics. Lu et al. [10] also applied both URANS and LES to calculate the cavitation based on a same unstructured grid. Baek et al. [11] investigated the wake feature of a propeller in open water in different advance ratios. They proposed an empirical model of $3 \mathrm{D}$ helices to provide an approach for propeller wake simulation. In these studies, the results show that the cavitation patterns on the blade can be predicted with a certain level of accuracy. However, the inhomogeneity of velocity components of the ship stern is not considered well. Francesco Salvatore and
Tom van [12] simulated unsteady propeller cavitation using CFD based on different turbulence models and discretization techniques and tested the performance of available computational tools for cavitation flow. However, the pressure of the noncavitation region on the blade is not predicted well. Kumar and Mahesh [13] simulated near and far wake evolution of a propeller and found a mutual-induction mechanism of instability between the tip vortices. Moreover, Bensow and Liefvendahl [14] used the large eddy simulation (LES) based on the explicit and implicit subgrid model to simulate the unsteady wake flow of a propeller. The prediction yielded fairly similar results and was close to the experimental data, but the flow structures diffuse quickly as a result of the grid resolution insufficiency. However, numerical simulation of propeller wake flow can indeed provide many complicated phenomena since many of the physics of the mass transfer are unknown. Moreover, the cavitation and vorticity dynamics are closely tied, both in time and space domain, necessitating more knowledge of propeller cavitation with the relative vorticity transport equation in the current study.

In the present paper, numerical simulation using the shear stress transport (k- $\omega S S T$ ) turbulence model combined with the Zwart-Gerber-Belamri (ZGB) cavitation model was conducted, inspired by previous investigations. By applying nonuniform inflow built by a ship wake at $J=0.6$ and $\sigma=5.5$ and 7.5, the cavitation flow is generated with a four-blade propeller, whose experimental data is abundant. Influence of mesh resolution was investigated using three refined structured meshes. Moreover, relationships between the cavitation and vorticity were investigated with the relative vorticity transport equation.

\section{Numerical Methods and Models}

2.1. Governing Equations. The present $3 \mathrm{D}$ flow calculation is governed by the continuity equation and momentum equation. Once the turbulence model based on the Reynolds averaging approach is applied, the equations can be written as

$$
\begin{aligned}
& \frac{\partial \rho_{m}}{\partial t}+\frac{\partial\left(\rho_{m} u_{j}\right)}{\partial x_{j}}=0 \\
& \frac{\partial\left(\rho_{m} u_{i}\right)}{\partial t}+\frac{\partial\left(\rho_{m} u_{i} u_{j}\right)}{\partial x_{j}}=\rho_{m} f_{i}-\frac{\partial p}{\partial x_{i}}+\frac{\partial}{\partial x_{j}}\left[\left(\mu_{m}+\mu_{t}\right)\left(\frac{\partial u_{i}}{\partial x_{j}}+\frac{\partial u_{j}}{\partial x_{i}}-\frac{2}{3} \frac{\partial u_{k}}{\partial x_{k}} \delta_{i j}\right)\right] .
\end{aligned}
$$

The two-phase flow is considered as the homogeneous and incompressible fluid so that the flow field shared the same velocity and pressure field. In the equations, $u_{i}(\mathrm{~m} / \mathrm{s})$ is the corresponding $i$ direction velocity component, $f_{i}(\mathrm{~N})$ is the body force in the $i$ direction, $\mu_{m}(\mathrm{~Pa} \cdot \mathrm{s})$ and $\mu_{t}(\mathrm{~Pa} \cdot \mathrm{s})$ are laminar viscosity and turbulent eddy viscosity, respectively, and $\rho_{m}\left(\mathrm{~kg} / \mathrm{m}^{3}\right)$ is the mixture density which can be modeled by the vapor volume fraction $\alpha_{v}$ :

$$
\rho_{m}=\rho_{v} \alpha_{v}+\rho_{l}\left(1-\alpha_{v}\right)
$$

where subscripts $v$ and $l$ denote vapor and liquid components, respectively. 
2.2. $k$ - $\omega$ SST Turbulence Model. According to a wide range of validated numerical studies, the $k$ - $\omega$ SST turbulence model developed from Menter's [15] work is believed to improve prediction of the flows with separation under the condition of adverse pressure gradients and flow structures around rotating machinery and therefore is adopted in the present study. The blending function $F_{1}$, used in the $k-\omega S S T$ turbulence model, is to blend the near-wall region and far field by $k-\omega$ and $k-\varepsilon$ model, respectively. The turbulent kinetic transport equations $k$ and the turbulent frequency $\omega$ are

$$
\begin{aligned}
& \frac{\partial(\rho k)}{\partial t}+\frac{\partial}{\partial x_{j}}\left(\rho u_{j} k\right)=\frac{\partial}{\partial x_{j}}\left[\left(\mu_{m}+\frac{\mu_{t}}{\sigma_{k 3}} \frac{\partial k}{\partial x_{j}}\right)\right]+P_{k}-\beta^{\prime} \rho k \omega, \\
& \frac{\partial(\rho \omega)}{\partial t}+\frac{\partial}{\partial x_{j}}\left(\rho u_{j} \omega\right)=\frac{\partial}{\partial x_{j}}\left[\left(\mu_{m}+\frac{\mu_{t}}{\sigma_{\omega 3}} \frac{\partial \omega}{\partial x_{j}}\right)\right]+\left(1-F_{1}\right) 2 \rho \frac{1}{\sigma_{\omega 3} \omega} \frac{\partial k}{\partial x_{j}} \frac{\partial \omega}{\partial x_{j}}+\alpha_{3} \frac{\omega}{k} P_{k}-\beta_{3} \rho \omega^{2},
\end{aligned}
$$

where $\alpha_{3}, \beta_{3}, \sigma_{\mathrm{k} 3}$, and $\sigma_{\omega 3}$ are of the corresponding coefficients for the $k-\omega$ and $k-\varepsilon$ models such as $\alpha_{3}$ :

$$
\alpha_{3}=F_{1} \alpha_{1}+\left(1-F_{1}\right) \alpha_{2} \text {. }
$$

The model coefficients are

$$
\begin{aligned}
\alpha_{1} & =5 / 9, \alpha_{2}=0.44, \beta_{1}=0.075, \beta_{2}=0.0828, \sigma_{k 1}=0.85 \\
\sigma_{k 2} & =1 . \sigma_{\omega 1}=2, \sigma_{\omega 2}=1 / 0.856, \beta^{\prime}=0.09
\end{aligned}
$$

The turbulence production is denoted as

$$
P_{k}=\mu_{t}\left(\frac{\partial u_{i}}{\partial x_{j}}+\frac{\partial u_{j}}{\partial x_{i}}\right) \frac{\partial u_{i}}{\partial x_{j}}-\frac{2}{3} \frac{\partial u_{k}}{\partial x_{k}}\left(3 \mu_{t} \frac{\partial u_{k}}{\partial x_{k}}+\rho k\right)
$$

The proper transport behavior is predicted by a restricted eddy viscosity as

$$
\mu_{t}=\rho \frac{a_{1} k}{\max \left(a_{1} \omega, \mathrm{SF}_{2}\right)},
$$

where $F_{2}$ is another blending function and $S$ denotes an invariant measure of the strain rate.

The blending functions $F_{1}$ and $F_{2}$ can be written in the following form:

$$
\begin{aligned}
F_{1} & =\tanh \left(\arg _{1}^{4}\right), \\
\arg _{1} & =\min \left(\max \left(\frac{\sqrt{k}}{\beta^{\prime} \omega y}, \frac{500 v}{\omega y^{2}}\right), \frac{4 \rho k}{C D_{k w} \sigma_{\omega 2} y^{2}}\right), \\
F_{2} & =\tanh \left(\arg _{2}^{2}\right), \\
\arg _{2} & =\max \left(\frac{2 \sqrt{k}}{\beta^{\prime} \omega y}, \frac{500 v}{\omega y^{2}}\right),
\end{aligned}
$$

where $y$ denotes the distance to the nearest wall and $v$ is the kinematic viscosity.

2.3. Cavitation Model. To simulate cavitation flows, the liquid and vapor phases need to be denoted in the simulation, as well as the transition mechanism between the two phases. The ZGB [16] cavitation model was adopted to capture the two-phase flow characteristics in the cavitation flow in the present study. The spatial and temporal alteration in the vapor volume fraction is illustrated by a transport equation including two source terms for the mass transfer between the two phases:

$$
\frac{\partial\left(\rho_{v} \alpha_{v}\right)}{\partial t}+\frac{\partial\left(\rho_{v} \alpha_{v} u_{j}\right)}{\partial x_{j}}=\dot{m}^{+}-\dot{m}^{-} .
$$

Based on equation (9), which is closed by the mass transfer rate in a bubble and the simplified Rayleigh-Plesset equation, the vaporization and condensation source terms are given as

$$
\begin{aligned}
& \dot{m}^{+}=F_{\text {vap }} \frac{3 \alpha_{\text {nuc }}\left(1-\alpha_{v}\right) \rho_{v}}{R} \sqrt{\frac{2}{3} \frac{\left|p_{v}-p\right|}{\rho_{l}}}, \\
& \dot{m}^{-}=F_{\text {cond }} \frac{3 \alpha_{v} \rho_{v}}{R} \sqrt{\frac{2}{3} \frac{\left|p_{v}-p\right|}{\rho_{l}}} .
\end{aligned}
$$

The model coefficients are

$$
R=1 \times 10^{-6} m, \alpha_{\text {nuc }}=2 \times 10^{-3}, F_{\text {vap }}=50, F_{\text {cond }}=0.01 \text {, }
$$

where $F_{\text {vap }}$ and $F_{\text {vap }}$ are empirical coefficients for phases' transfer process, $\alpha_{\text {nuc }}$ is the noncondensable gas fraction in liquid, and $R$ is the typical bubble size in liquid.

\subsection{Propeller Geometry, Meshing Technique, and Boundary} Conditions. The propeller INSEAN E779A is an old designed four-bladed propeller, but a large number of experiments and research studies have been conducted with it. The database set covers both uniform as well as nonuniform inflow for both cavitation and noncavitation conditions. The propeller model is shown in Figure 1, and the propeller diameter is $D=0.227 \mathrm{~m}$. In order to avoid the effect of relative motion between the rotating and the external domain, the stationary domain was simplified to a cylinder extending $0.3 D$ upstream the propeller plane and $5 D$ downstream with a radius of $3 D$. For the nonuniform inflow condition, refinement in the propeller downstream wake flow was sacrificed in order to raise the resolution upstream the blade, in order to transport the inhomogeneous wake 


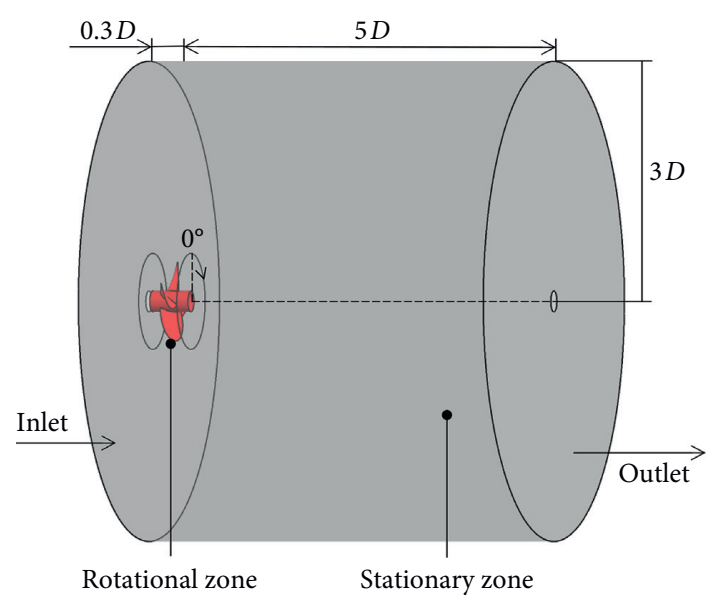

Figure 1: Computational domain.

more accurately. The distance of $0.3 D$ is selected based on a large number of comparisons. The computational domains are totally composed of hexahedral grids and have been manually refined in the blade boundary layer, cavitation region, and the propeller upstream, as shown in Figure 2. The same systematic topology is used to produce three sets of refined grids with a refinement ratio of $\sqrt{2}$, as shown in Table 1.

We have simulated the unsteady cavitation flow around the propeller under two conditions. A highly nonuniform wake field was applied to generate a dynamic loading variation of the propeller, which consequently forms an unsteady cavity evolution, by referencing experimental results reported by Pereira et al. [17]. The inlet boundary was defined as an inhomogeneous inflow condition and the outlet was the pressure outlet boundary. The outer wall of the stationary domain was modeled as the free slip wall and the propeller and hub surfaces were all regarded as no-slip walls. The outlet pressure was obtained with the cavitation number $\sigma_{n}=\left(p_{\text {out }}-p_{v}\right)$ / $\left(0.5 \rho_{l} \pi n^{2} D^{2}\right)=5.5,7.5$. The value of the Reynolds number $\operatorname{Re}_{0.7 \mathrm{R}}=C_{0.7 R} \sqrt{U_{\infty}^{2}+\left(0.7 \pi n D_{m}\right)^{2}} / v=1.8 \times 10^{6}$

To investigate the unsteady cavitation evolution on the propeller, the convergent noncavitation results was regarded as initial data to solve the unsteady cavitation calculation. The time-dependent governing equations were solved by the spatial and temporal discretization method. The advection term used in the governing equations is the high resolution with the central difference scheme applied for the diffusion terms and unsteady second order implicit formation for the unsteady terms. $10^{\circ}$ per time step was adopted for the first 5 cycles in the unsteady calculation and then $1^{\circ}$ per time step for the next 15 cycles. These first 10 revolutions were employed to remove the effect of the initial results, and 10 more revolutions continued with $1^{\circ}$ per time step have been kept to get the results put forward in the following analysis.

\section{Results and Discussion}

3.1. Influences of Mesh Resolution. In order to emulate a nonuniform ship wake, a velocity deficit region is created in the inflow of the propeller as disturbed by the ship wake. The inlet velocity distribution affects the propeller cavitation significantly. The nonuniform wake fields predicted with three meshes are shown in Figure 3. Figure 3 shows dimensionless velocity $\left(u / U_{\infty}\right)$ distribution contours on the stationary and rotating interfaces. The nonuniform velocity fields predicted by different meshes not only are similar in general but also show some distincts in the meanwhile. With more refined meshes, the wake field presents more details. The nonuniform flow characteristics are satisfactorily reproduced and the high consistency between the rotating and stationary interface shows that the velocity can be efficiently transmitted downstream. Transient cavitation is generated on the propeller when the blade sweeps the velocity deficit region, and the results show that the predicted cavitation in Figure 4 becomes more unstable.

To validate the simulation result of unsteady cavitation, Figure 4 displays the development of cavitation on propeller blade predicted with different meshes at three typical moments. The cavitation was visualized with the iso-surface of vapor volume fraction $\alpha_{v}=0.1$ and was compared with the experimental results, which provide the cavitation snapshots for $\theta$ from $-20^{\circ}$ to $20^{\circ}$ with increments of $20^{\circ}\left(\theta=0^{\circ}\right.$ matching

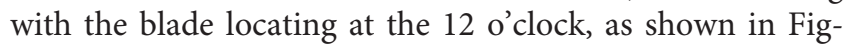
ure 1). When the propeller passes through the velocity deficit region at the blade position of $-20^{\circ}$, the cavitation starts to produce from the leading edge. From $-20^{\circ}$ to $20^{\circ}$, the cavity grows up and gradually rolls up to the blade tip. The cavity on the leading edge is observed to be cut off by the sideentrant jet, which is produced by the shedding cavity in the trailing region. Comparison between the predictions and the experimental snapshots shows that the cavitation patterns are fairly well reproduced by the current method, even though there is a little underestimation of the cavitation in the tip vortex region of the blade tip. Moreover, the predicted unstable cavitation is closer to experiments with the more refined mesh. The investigations show that Mesh 2 can well capture the cavitation flow features around the propeller and also balance the numerical accuracy and time. Therefore, the predicted results by Mesh 2 are employed in the following discussion.

\subsection{Analysis of the Unsteady Propeller Cavitation.} Cavitation results of both experiment and simulation are recorded for specific cavitation numbers $\sigma$, which equal to 7.5 and 5.5. Figure 5 displays the cavitation pattern for $\sigma=5.5$ and 7.5 against position angles from $-20^{\circ}$ to $20^{\circ}$ with the snapshots angular step of $10^{\circ}$, as the blade sweeps through the turbulent wake. It is indicated that leading edge cavity occurs around $-20^{\circ}$, and the cavitation inception and initial expansion are captured in two conditions. The cavity grows up as the blade closes to the vertical position where it is in the wake field and reaches a maximum at about the $10^{\circ}$ position. The cavitation attached within a wide span of leading edge, and subsequently, the cavity gradually shrinks from the blade root to the tip as the blade passes through the wake region. As the cavitation number increases to 7.5, an obvious cavity disruption appears at $\theta=20^{\circ}$, which is linked 

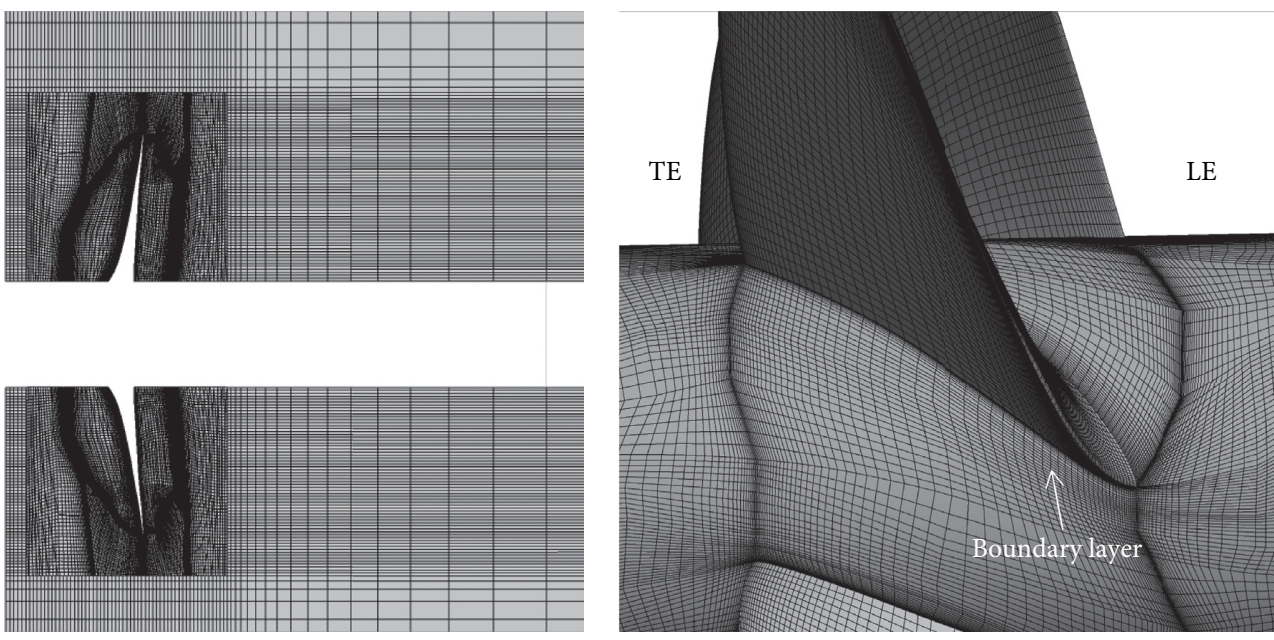

(a)

(b)

Figure 2: Grids along the propeller surface for Mesh 2.

TABLE 1: Details of three systematically refined meshes.

\begin{tabular}{lccc}
\hline Elements' number & Mesh 1 & Mesh 2 & Mesh 3 \\
\hline Rotational zone & 678368 & 2095104 & 6148688 \\
Stationary zone & 462720 & 1009824 & 2959488 \\
Total & 1141088 & 3104928 & 9108176 \\
\hline
\end{tabular}

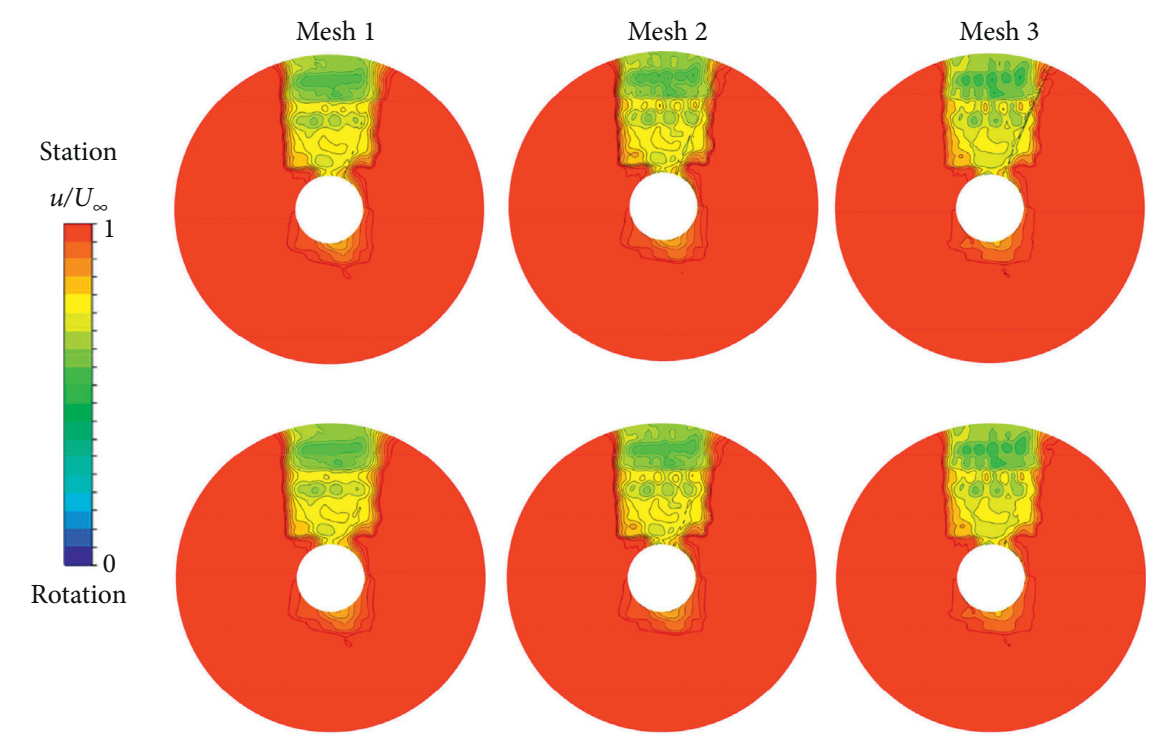

FIgURE 3: Comparison of axial velocity component at the stationary and rotating interfaces.

to the side-entrant jet generated from the leading edge of the blade. In this moment, the sheet cavity is entirely developed with side-entrant jet along the trailing region of the cavity rolling up into the tip vortical structure. It is shown that the larger part of the cavity is separated from the blade surface caused by the side-entrant jet and transformed into a small amount of cloud cavities. The abovementioned typical characteristics, which can significantly influence the evolution of cavitation, are more obvious in the condition of $\sigma=7.5$.

3.3. Cavitation Flow Structures around the Propeller. In Figure 5, the sheet cavitation is gradually developed as the side-entrant jets roll up a larger part of the cavity into the tip. And, it is noted that the trailing part of the cavitation is fairly 

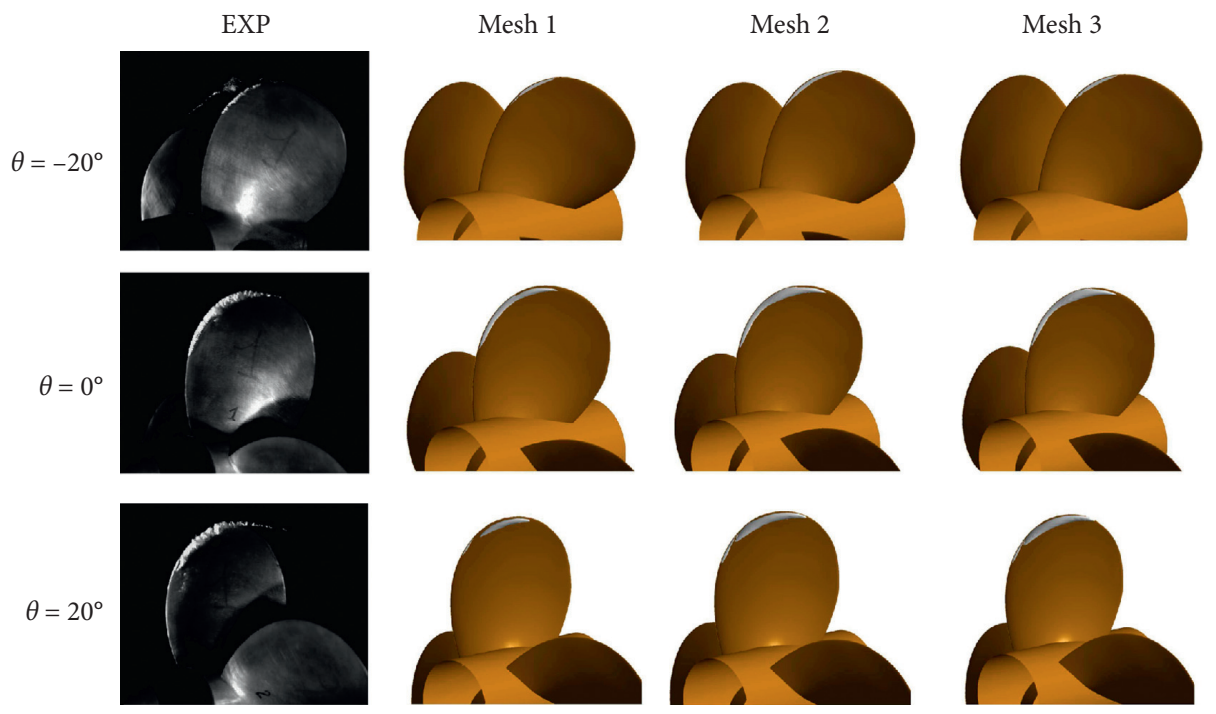

Figure 4: Comparison of the simulated and measured cavitation patterns as the propeller rotation by the three set of meshes [1, 17].

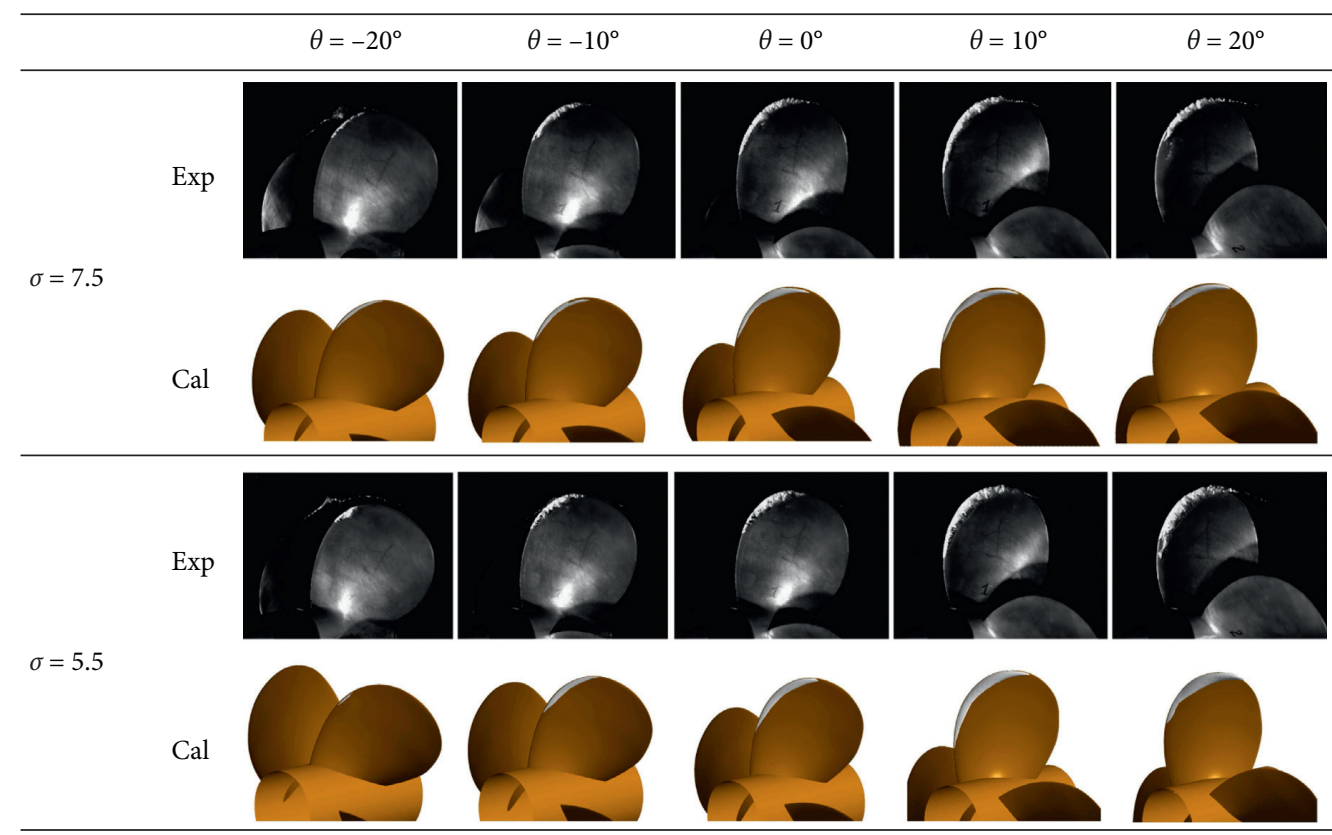

FIGURE 5: Development of the cavity on the propeller in two different cavitation numbers $(\sigma=5.5 ; \sigma=7.5)[1,17]$.

apart from the blade surface due to the side-entrant jet and transforms into cloud cavities. At the instant of $\theta=20^{\circ}$, the numerical result is accurately predicted as compared with what was indicated by the experimental snapshots, and the side-entrant jets are clearly visible, which bring liquid between the sheet cavitation and the blade surface and move into the tip vortex. Overall, the computed cavities are similar with the experiments, for both tip cavity on the blade tip and the sheet cavity at the leading edge, which are predicted at the correct locations and with accurate shapes.

The feature of the leading edge flow is studied in Figure 6 in detail. It is indicated that typical flow features in the snapshots from the experiments under the condition of $\sigma=7.5$ are all captured by the simulation results. In the second figure, the cavitation is fully developed and the blade surface detachment has started. The cavitation pattern and streamline on the blade surface are shown, as well as the velocity field around the blade tip. It shows that once the side-entrant jets generate, a vortical structure is created, which will greatly influence the dynamic behavior of the trailing part of the cavity. Such a vortex flow is common on marine propellers and can be exacerbated with the highly nonuniform wake applied in the present study. Usually the tip vortex appears just at the blade tip owing to the flood from the pressure side to the suction side of the blade, but the vortical structure also develops around the trailing part of the cavity because of the side-entrant jet. In the first figure, it is obvious that the cavitation has still not extended to the 


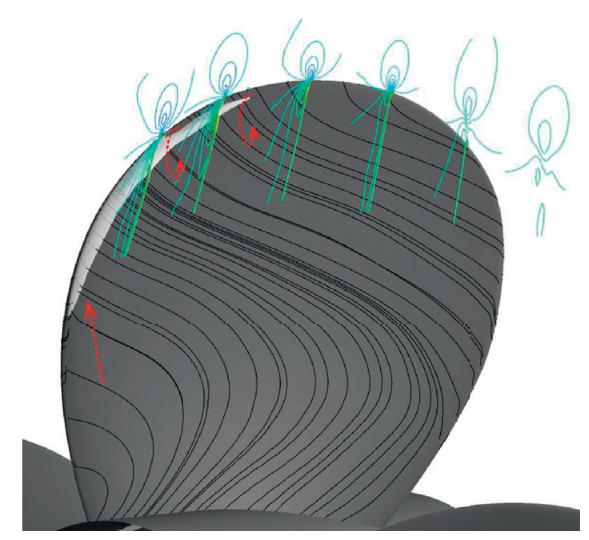

(a)

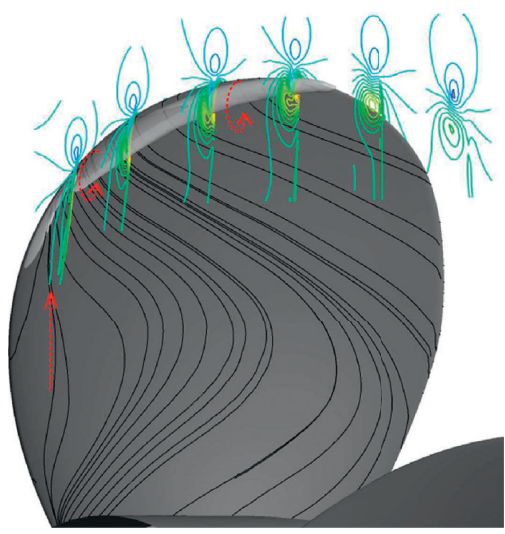

(b)

Figure 6: The cavity pattern is shown by an iso-surface of vapor volume fraction $\alpha_{v}=0.1$ at two instants, complemented by velocity contours of the flow around the cavitation and blade tip.

tip. Presence of the interaction between the generated vortical structure and the cavity is an unsteady and complex phenomenon. Studying and understanding vortex dynamic features, as is to some extent displayed in the present investigation, are a significant step toward improved propeller designs.

3.4. Propeller Cavitation Influences on the Vorticity Distribution. The available experimental data provide primary results, including a number of snapshots, and measures of cavity extent. However, some potential flow mechanisms, which are crucial and should be obtained from detailed vortical structures as well as interaction between cavitation and vortex, can only be obtained with the simulation. The vorticity has been proved to be strongly affected by the complex transient cavitation around hydrofoils [18-20] with the vorticity transport equation. The impact of the cavitation around a marine propeller shown in this study on the vorticity distribution is also investigated by the relative vorticity transport equation [21-23]:

$$
\begin{aligned}
\frac{\mathrm{d} \vec{\omega}}{\mathrm{d} t}= & (\vec{\omega} \cdot \nabla) \cdot \vec{u}-\vec{\omega} \cdot(\nabla \cdot \vec{u})+\frac{1}{\rho^{2}} \nabla \rho \times \nabla p+v \nabla^{2} \vec{\omega} \\
& -2 \nabla \times\left(\omega_{1} \times \vec{u}\right),
\end{aligned}
$$

where $\omega$ indicates the relative vorticity, $u$ is the relative velocity, $\nu$ is the kinematic viscosity, and $\rho$ is the mixture density.

In equation (12), the term on the left-hand side is the rate of vorticity change due to the fluid convection. On the righthand side, the first term denotes a vortex stretching and tilting that is mainly attributed to velocity gradients. The second term is the relative vortex dilation that is attributed to the effects of fluid volumetric expansion or contraction on relative vorticity. The third term represents baroclinic torque that results from the pressure and mixture density gradients misalignment. The fourth term originates from viscous diffusion and can result in the change of relative vorticity. The last term denotes the effects of the Coriolis force, which plays an important role in relative vorticity distribution in the rotational frame.

To visualize the influence of cavitation on vortex distribution around the propeller, the simulated results are demonstrated on the suction side in Figure 7, along with the contours of vapor volume fraction $\alpha_{v}=0.1$, relative vorticity, and all terms of equation (1) at two typical instants $\left(\theta=-10^{\circ}\right.$ and $\left.20^{\circ}\right)$. Figure 7 shows a sketch of planes S1 S6 and S7 S12 in two moments. Flow features are obviously observed along the blade suction side. Contours of the vapor volume fraction and relative vorticity are shown in Figures 7(a) and 7(b), respectively, and the relative vorticity mainly concentrated in the cavitation region and in the cavity shedding wake, which demonstrate that vorticity intensity is closely linked to the cavitation development. Figure $7(\mathrm{c})$ clearly indicates that the effect of vortex stretching term is more important for vortex distribution during cavitation development than other terms in the blade tip, along the cavity interface and in the downstream cavity shedding region. The side-entrant jet transports a significant amount of the vortex stretching term from outside the cavity to inside when the cavity attaches the blade surface, as shown on S1 S3 and S7 S8. The strong tip cavity, separated from blade surface, shows important influence on the vortex stretching term distribution on S4. As shown on S4 and S8 in Figure 7 (c), the cavitation avoids the pressure decrease in the core and inhibits the vortex generation. However, the highly irregular processes of mass transfer along the cavity shedding region promote the vortical structure generation and unsteady turbulence on S5 S6 and S10 S12.

Moreover, magnitudes of vortex dilatation are obviously larger along the cavity surface than that which occurs in the cavity core, which is directly linked to the velocity divergence. Note that the baroclinic torque term mainly distributes in the cavitation region because density gradients misalign with pressure gradients in the cavitation flow. Effects of the baroclinic torque are mainly along the liquidvapor interface and the staggered arrangement of the term 

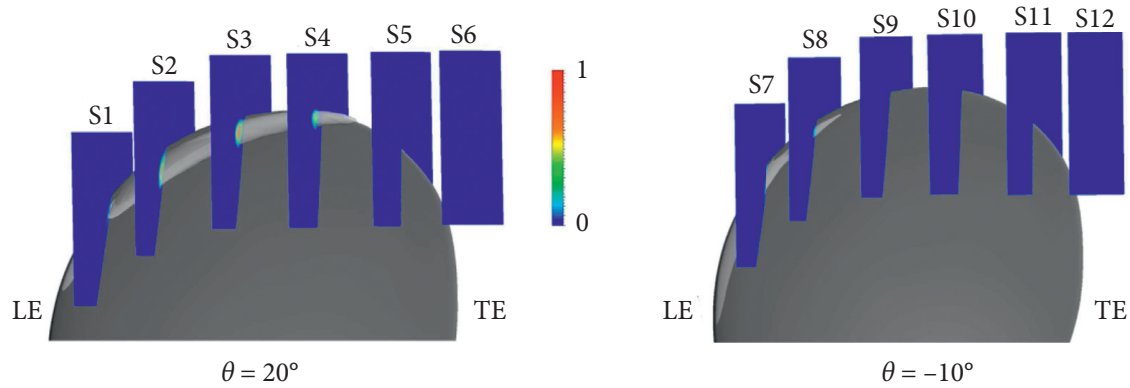

(a)
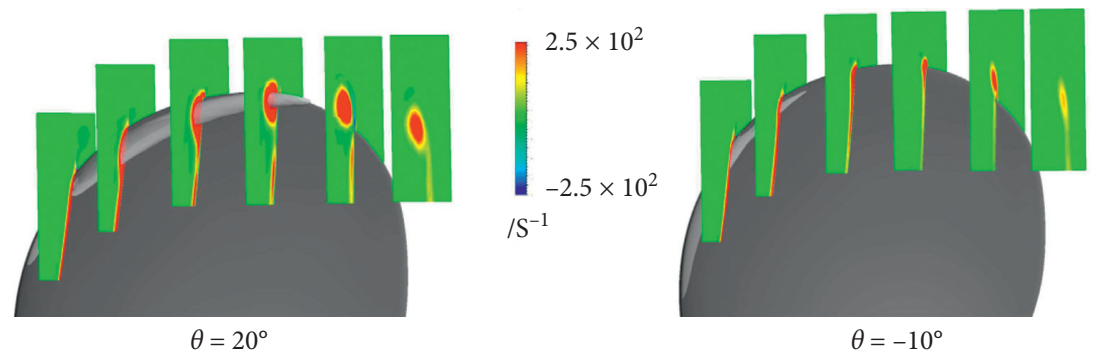

(b)
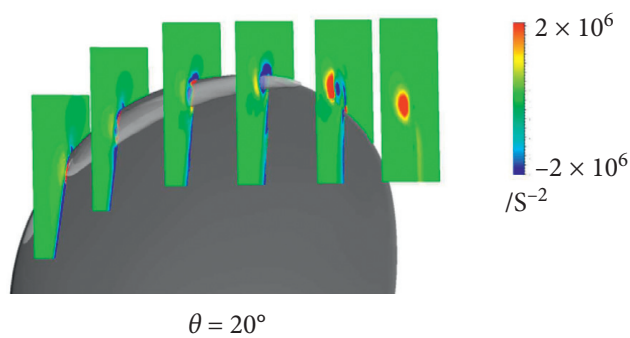
${ } \mathrm{S}^{-2}$

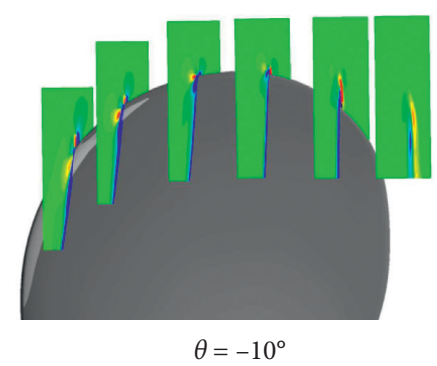

(c)
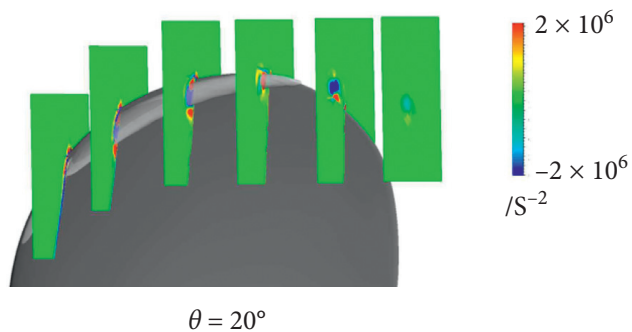
$/ \mathrm{S}^{-2}$

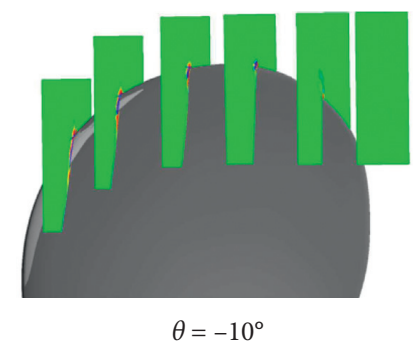

(d)
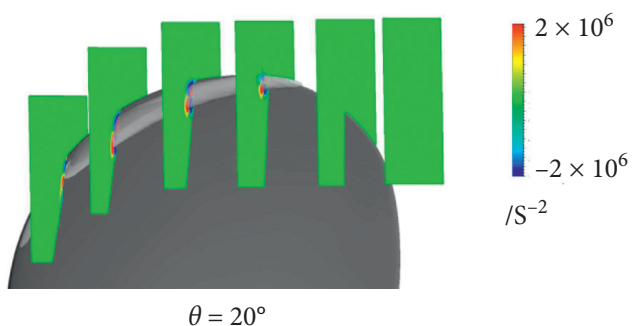
$/ \mathrm{S}^{-2}$

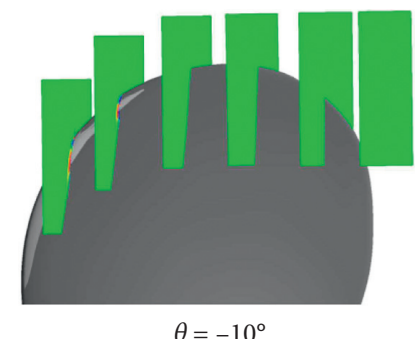

(e)

Figure 7: Continued. 


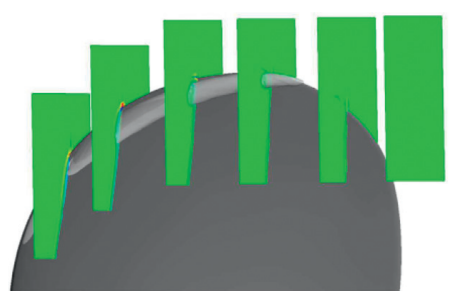

$\theta=20^{\circ}$

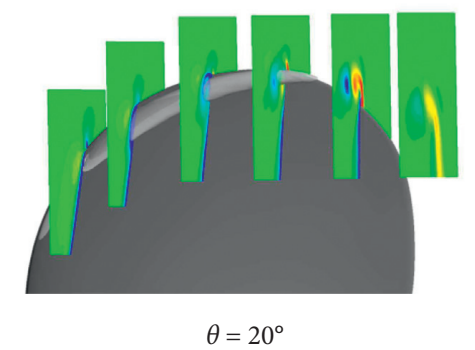

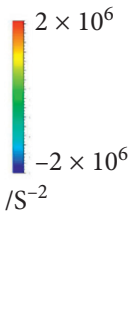

(f)

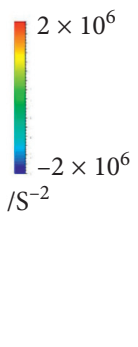

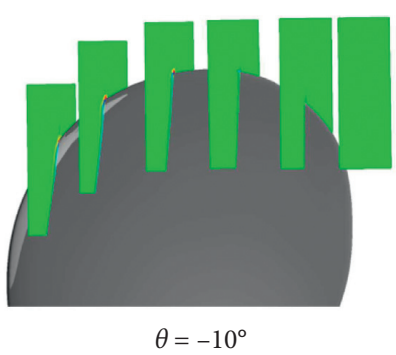

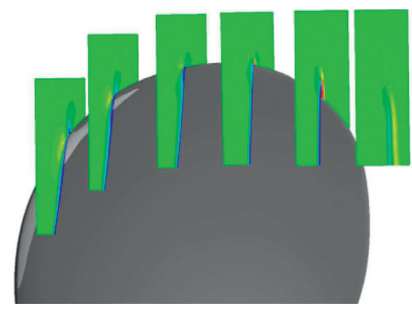

$\theta=-10^{\circ}$

(g)

FIgURe 7: Contours of the predicted vapor volume fraction, relative vorticity, vortex stretching, dilatation, baroclinic torque, and Coriolis force terms on $\mathrm{S} 1$ to $\mathrm{S} 12$.

magnitude promotes the vorticity reallocation. Although the baroclinic torque term shows remarkable effects on cavitation, the major source of vortex production is the dilation term, whose intensity appears to be greater when compared with that of the baroclinic torque term. The Coriolis force term effects should be considered in rotational machines, and the distributions of the term show the same tendency with the stretching term, while it is smaller around the cavity. The viscous diffusion term is not much visible and can be disregarded compared with the other terms.

\section{Conclusions}

Turbulent cavitation flows around a marine propeller were studied using the $k-\omega S S T$ turbulence model and the ZGB cavitation model. The following conclusions can be drawn from the present investigation:

(1) The calculated cavitation characteristics in two conditions predicted by the current numerical method match reasonably well with the experimental results. The wake fields predicted by the three refined grids are very nonuniform and the cavity patterns have a high agreement with experiment in both space and time domain. The study has been carried out using both experimental and simulated techniques, yielding complementary databases.

(2) It is significant that the main cavitation flow behavior, such as side-entrant jet and cavitation evolution, are captured by the numerical simulation in order to progress toward reliable predictive techniques. As the sheet cavitation is fully expanded, the side-entrant jet moves along the trailing part of the cavity and rolls up into the blade tip. And, then, the cavity detaches from the blade surface caused by the side-entrant jet. The vorticity distribution is also fairly effected by the sideentrant jet around the cavitation.

(3) Analyses based on the relative vorticity transport equation suggest that cavitation shows great effects on the vorticity distribution around the propeller. The relative vorticity mainly concentrates around the cavity and the shedding wake. The stretching term is the main contributor to vortex generation, whose effect appears to be more important when compared with the other terms. Although the vortex dilation term and baroclinic torque term play an influential role in cavitation-vortex flow, the vortex dilation term has more significant effect than the baroclinic torque. The effects of the Coriolis force should be considered around the propeller flow, and the viscous diffusion term is quite small and can be neglected.

\section{Data Availability}

Previously reported experimental data were used to support this study and are available at https://doi.org/10.3390/ jmse4040070. These prior studies (and datasets) are cited at relevant places within the text as references [1] and [17].

\section{Conflicts of Interest}

The authors declare that there are no conflicts of interest regarding the publication of this paper.

\section{Acknowledgments}

The authors would like to appreciate Dr. Francesco Salvatore and Dr. Francisco Alves Pereira for opening geometry of the 
INSEAN E779A marine propeller and the experimental data. This work was financially supported by the National Natural Science Foundation of China (Project nos. 11772239 and 51822903). The numerical calculations in this study have been done on the supercomputing system in the Supercomputing Center of Wuhan University.

\section{References}

[1] F. Alves Pereira, F. Di Felice, and F. Salvatore, "Propeller cavitation in non-uniform flow and correlation with the near pressure field," Journal of Marine Science and Engineering, vol. 4, no. 4, p. 70, 2016.

[2] T. Ito, "An investigation into the unsteady cavitation of marine propellers," Journal of Zosen Kiokai, vol. 1962, no. 111, pp. 137-145, 2009.

[3] G. Bark and W. B. Van Berlekom, "Experimental investigations of cavitation noise," in Proceedings of the 12th ONR Symposium on Naval Hydrodynamics, pp. 470-493, Washington, DC, USA, 1978.

[4] A. Stella, G. Guj, and F. Di Felice, "Propeller wake flowfield analysis by means of LDV phase sampling techniques," $E x$ periments in Fluids, vol. 28, no. 1, pp. 1-10, 2000.

[5] P.F. Andrea, "An improved wake description by higher order velocity statistical moments for single screw vessel," Ocean Engineering, vol. 108, pp. 181-190, 2015.

[6] S. J. Lee, B. G. Paik, J. H. Yoon, and C. M. Lee, "Threecomponent velocity field measurements of propeller wake using a stereoscopic PIV technique," Experiments in Fluids, vol. 36, no. 4, pp. 575-585, 2004.

[7] M. Felli, F. Di Felice, G. Guj, and R. Camussi, "Analysis of the propeller wake evolution by pressure and velocity phase measurements," Experiments in Fluids, vol. 41, no. 3, pp. 441-451, 2006.

[8] J. W. Lindau, D. A. Boger, R. B. Medvitz, and R. F. Kunz, "Propeller cavitation breakdown analysis," Journal of Fluids Engineering, vol. 127, no. 5, pp. 995-1002, 2005.

[9] W. Cai, Y. Li, and C. Liu, "Comparative study of scaleresolving simulations for marine-propeller unsteady flows," International Communications in Heat and Mass Transfer, vol. 100, pp. 1-11, 2019.

[10] N.-X. Lu, R. E. Bensow, and G. Bark, "Large eddy simulation of cavitation development on highly skewed propellers," Journal of Marine Science and Technology, vol. 19, no. 2, pp. 197-214, 2014.

[11] D.-G. Baek, H.-S. Yoon, J.-H. Jung, K.-S. Kim, and B.-G. Paik, "Effects of the advance ratio on the evolution of a propeller wake," Computers \& Fluids, vol. 118, pp. 32-43, 2015.

[12] H. S. Francesco Salvatore and T. Tom van, "Propeller cavitation modelling by CFD -results from the VIRTUE 2008 rome workshop," in Proceedings of the First International Symposium on Marine Propulsors 2009, Trondheim, Norway, June 2009.

[13] P. Kumar and K. Mahesh, "Large eddy simulation of propeller wake instabilities," Journal of Fluid Mechanics, vol. 814, pp. 361-396, 2017.

[14] R. Bensow and M. Liefvendahl, "Implicit and explicit subgrid modeling in les applied to a marine propeller," Fluid Dynamics Conference \& Exhibit2008, vol. 3, pp. 1511-1522, 2008.

[15] F. R. Menter, "Two-equation eddy-viscosity turbulence models for engineering applications," AIAA Journal, vol. 32, no. 8, pp. 1598-1605, 1994.

[16] P. J. Zwart, A. G. Gerber, and T. Belamri, "A Two-phase Flow Model for Predicting Cavitation Dynamics," in Proceedings of the ICMF 2004 International Conference on Multiphase Flow, Yokohama, Japan, May 2004.

[17] F. J. A. Pereira, F. Salvatore, F. Di Felice, and M. Soave, "Experimental investigation of a cavitating propeller in nonuniform inflow," in Proceedings of the 25th Symposium on Naval Hydrodynamics 2004, Labrabor, Canada, June 2004.

[18] C. Z. Han, Y. Long, B. Ji et al., "An integral calculation approach for numerical simulation of cavitating flow around a marine propeller behind the ship hull," Journal of Hydrodynamics, vol. 30, no. 1, pp. 1186-1189, 2018.

[19] X. P Long, Y. LongW. T. Wang et al., Some notes on numerical simulation and error analyses of the attached turbulent cavitating flow by LES," Journal of Hydrodynamics, vol. 30, no. ., pp. 1-4, 2018.

[20] H. Y. Cheng, X. R. Bai, X. P. Long, B. Ji, and M. Farhat, "Large Eddy Simulation of the Tip-leakage Cavitating flow with an insight on how cavitation influences vorticity and turbulence," Applied Mathematical Modelling, vol. 77, pp. 788-809, 2019.

[21] R. Huang, B. Ji, X. Luo, Z. Zhai, and J. Zhou, "Numerical investigation of cavitation-vortex interaction in a mixed-flow waterjet pump," Journal of Mechanical Science and Technology, vol. 29, no. 9, pp. 3707-3716, 2015.

[22] Y. Long, X. Long, B. Ji, and H. Huang, "Numerical simulations of cavitating turbulent flow around a marine propeller behind the hull with analyses of the vorticity distribution and particle tracks," Ocean Engineering, vol. 189, p. 106310, 2019.

[23] C.-z. Han, S. Xu, H.-y. Cheng, B. Ji, and Z.-y. Zhang, "LES method of the tip clearance vortex cavitation in a propelling pump with special emphasis on the cavitation-vortex interaction," Journal of Hydrodynamics, vol. 32, no. 6, pp. 1212-1216, 2020. 